\title{
A comparative analysis of the stress-strain state of disc specimens in assessing the structural strength of materials
}

\author{
L.B. Tsvik ${ }^{\mathrm{a}}$ and E.V. Zenkova, ${ }^{\mathrm{a}} \mathrm{b}^{*}$
}

${ }^{a}$ Department of Carriages and Carriage Facilities, Irkutsk State Transport University, 15, Chernishevskogo, Irkutsk, 664074, Russian Federation ${ }^{b}$ Department of Mechanics and Strength of Materials, Irkutsk National Research Technical University, 83, Lermontov, Ir-kutsk, 664074, Russian Federation

A R T I C L E I N F O

Article history:

Received 20 July 2021

Accepted 6 December 2021

Available online

6 December 2021

Keywords:

Stress-strain state (SSS)

Disc specimen

Structural strength of material

Geometrical pa-rameters

Numerical modeling

Limit state equation

\section{A B S T R A C T}

A study of the stress-strain state (SSS) of disc specimens has been conducted when test-ing specimens with stress concentrators (grooves). The research has shown that the trun-cation of circular discs along two symmetrical chords makes it possible to change the type (the ratio of principal stresses) of SSS that occurs at the destruction site. Therefore, these specimens can be used to assess the structural strength of materials on standard single-drive testing machines, taking into account the real type of SSS that occurs in the bearing elements of machines. The results of numerical SSS modeling have been used to build the dependences of the SSS type and level on geometric parameters of specimens. Geometrical parameters can be chosen for a certain SSS type to assess both the static and fatigue strengths of materials used in the manufacture of bearing elements.

\section{Introduction}

Bearing structural elements experience static or cyclic loads that create a volumetric stress-strain state (SSS) as a concentration of stress near the structural irregularities (holes, protrusions, grooves, etc.) with destruction sites. The SSS affects the service life of structures under the static and cyclic loading (Kogaev et al. 1985). Under the compression, uniaxial tension or shear, the material strength criteria can be different for different SSS types (Smirnov-Alyaev, 1968; Tsvik et al., 1978), which requires to use unified strength criteria and various limit state equations (Kogaev et al. 1985; Pisarenko et al.; 2008; Lebedev et al.,2003; Tsvik, \& Zenkov, 2015a,b, 2018; Zenkov, 2017).

The static structural strength with regard to the SSS type can be calculated by various limit state equations (e.g., PisarenkoLebedev's equation, Yagn-Buzhinsky's equation, Drucker-Prager's equation) (Kogaev et al. 1985). In Zenkov \& Tsvik, (2017, 2018); Zenkov (2017), the computational and experimental method for calculating the structural strength has been described. It can be used to calculate the refined strength characteristics (values of the weight factors for the components responsible for fracture mechanisms) that are determined by destroying the specimens that have the SSS type similar to the one in the structure under study. The specimens can be tested to destruction on standard single-drive testing machines (Tsvik, 2017 ; Tsvik et al. 2020). The method can be used on special testing equipment with several power drives that creates multidirectional effects on the specimen (Zenkov \& Tsvik, 2018). However, the process of testing is difficult (Zenkov \& Tsvik 2018; Zenkov, 2017; Tsvik, 2017; Tsvik et al. 2020).

\footnotetext{
* Corresponding author.

E-mail addresses: jovanny1@yandex.ru (E.V.Zenkov) 
The SSS-based method involves the following stages:

- Calculation of the SSS level and type under conditions similar to the operation ones;

- Determination of SSS invariants included in the combined strength criterion of the structural material at possible destruction sites;

- Selection of laboratory specimens for testing to destruction at a given (selected by the results of preliminary calculation of SSS characteristics) value of SSS invariants that determine the strength in accordance with the combined strength criterion.

At the first stage, computer engineering technologies are used. The finite element method (FEM) is applied in solving problems of solid mechanics. The FEM-based apparatus of deformation mechanics and numerical and analytical methods for solving boundary and contact problems of mechanics are used to calculate the stress-strain state in MSC.PATRAN, ANSYS, Femap (with NX Nastran), etc. The second task can be solved in an automated mode by calculating the function set in the software. At this stage, characteristics of the SSS are determined (Zenkov et al. 2019). In accordance with the method described in (Zenkov \& Tsvik, 2018; Zenkov, 2017; Tsvik, 2017), the third problem can be solved if there are laboratory specimens appropriate for mechanical testing. During the destruction, their SSS invariants (SSS type) coincide with the corresponding values of the SSS characteristics determined at the second stage. $P$ is a criterion of similarity of the SSS that occurs in the structures and corresponding specimens. It is the ratio of the first stress tensor invariant to the second one (Kogaev et al. 1985; Smirnov-Alyaev, 1968; Pisarenko et al., 2008),

$$
P=\frac{\sigma_{1}+\sigma_{2}+\sigma_{3}}{\sigma_{i}},
$$

where $\sigma_{1}, \sigma_{2}$ and $\sigma_{3}$-stresses at the destruction site; $\sigma_{i}$ - equivalent stress calculated by formula,

$$
\sigma_{i}=\frac{1}{\sqrt{2}} \sqrt{\left(\sigma_{1}-\sigma_{2}\right)^{2}+\left(\sigma_{1}-\sigma_{3}\right)^{2}+\left(\sigma_{2}-\sigma_{3}\right)^{2}}
$$

Strain measurement and numerical modeling of stress fields of highly loaded elements of machines and mechanisms have shown that the values of $P$ at the destruction site are in the range of,

$-2 \leq P \leq-1 ; \quad 1 \leq P \leq 2$

The calculation of strength in the contact zone of the rolling element of the railway car axle box bearing is an example of determination of the SSS $(P<0)$. In this case, $P$ is close to -2 ; for ShKh4 steel used in the manufacture of bearings, the permissible contact stress is $3500 \mathrm{MPa}$ (GosNIIV 1996), which is double the ultimate strength of this steel under the uniaxial tension (1250 MPa). Experiments that have revealed a decrease in the strength under the biaxial tension (the hard stress-strain state type corresponding to the second in inequalities (3) (Smirnov-Alyaev, 1968) are described in (Zenkov \& Tsvik, 2015; Vilimok et al., 2013).

When calculating the structural strength using the combined strength criteria, in laboratory specimens, it is difficult to create a SSS type with a different ratio of the SSS invariants in accordance with inequalities (3). At $P=2$, the ratio of SSS invariants corresponding to the hard SSS type is most dangerous.

\section{Analysis of computational stress-strain state modeling of existing disc specimens}

Currently, to assess the strength under complex stress-strain conditions, specimens of various shapes (e.g., laboratory disctype specimens) can be used (e.g., a disc specimen with stress concentrators - U- and V-shaped notches of the same size and configuration located on the narrow opposite sides (edges) of the disc (GOST 25.504-82, 2004).

The use of such specimens does not ensure the required accuracy of calculation of the structural strength, since the variation in $P$ in the range corresponding to inequalities (3) is limited. The specimen of a prismatic shape with longitudinal and transverse planes of symmetry and two lateral protrusions located longitudinally can improve the accuracy of SSS simulation (Zenkov et al., 2014). The specimen has a U-shaped cross-section. Under the uniaxial force in the working zone located on the surface of the fillet transition in the middle part of the specimen adjacent to its transverse plane, it creates a stress-strain state type that satisfies one of inequalities (3). A significant drawback of this specimen is its complex shape (a system of grooves and protrusions) and a scheme of location in the bearing elements, which requires the use of both massive lateral prismatic supports and two end supports, which raises the mating surface manufacture accuracy and quality requirements for creating the biaxial SSS. 
To assess the strength of materials under the complex stress state (Fig. 1), let us study the laboratory specimen (Tsvik et al., 2009) of a round disc shape with stress concentrators (U- or V-shaped grooves) located on the opposite sides of the disc (a disc specimen). When testing the disc specimen, its support is used along the outer edge and in its working zone located in the center of a circular disc on the surface of the lower U-shaped groove. The stress-strain state can satisfy one of inequalities (3).

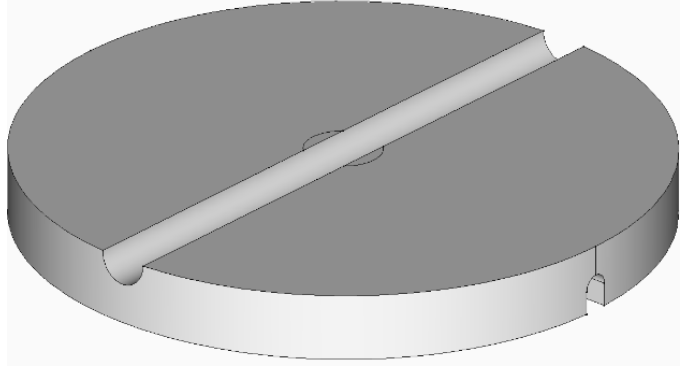

a

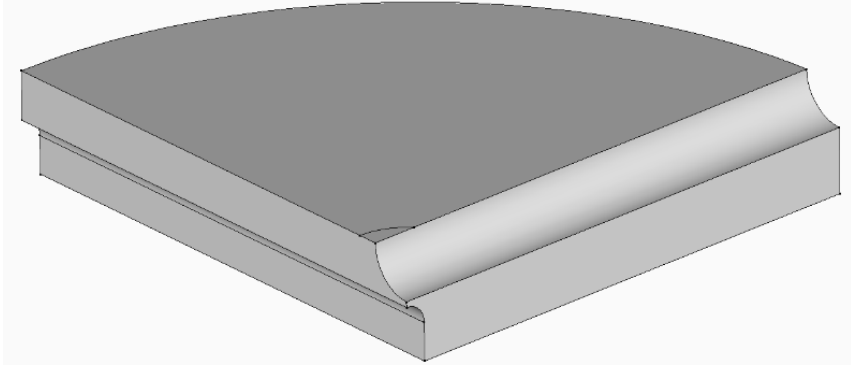

b

Fig. 1. Structural shape of the disc specimen

(a) General view and (b) A quarter of the specimen truncated by two planes of symmetry

Fig. 1(b) shows that in the vicinity of the axis of rotation of the original circular disc where the grooves are located, the material forms a relatively thin layer of a variable thickness between the groove surfaces. In mechanical testing, the specimen is supported along its outer circular contour (in Fig. 1, the lower side of the circular disc is bearing). In its central part, the specimen is loaded with a vertical downward force (perpendicular to the median surface of the circular disc). The force is distributed on the load side of the specimen over the small circular surface (the circular sector in Fig. 1).

\section{Mathematical model of deformation of disc specimens}

To study the dependences of variation in the SSS type, the mathematical model of volumetric elastic deformation has been built. For homogeneous isotropic materials, the differential equilibrium equations of the theory of elasticity $\mathbf{u}$ are used,

$$
L \mathbf{u}=(\lambda+\mu) \operatorname{grad} \operatorname{div} \mathbf{u}+\mu \Delta \mathbf{u}=0
$$

where $L$ - differential operator of the theory of elasticity; $\boldsymbol{u}$ - target displacement vector at points of region D occupied by the deformable elements; $\lambda$, $\mu$-coefficients that determine the mechanical properties of a homogeneous isotropic material (Lame parameters). Eq. (4) is applied to the region occupied by the material and the bounded surface $S=S_{\sigma}+S_{u \sigma}$, under boundary conditions,

$$
\left.(v \cdot \mathbf{T})\right|_{\mathrm{S}_{\sigma}}=\mathbf{F}(M), M \in \mathrm{S}_{\sigma} ;\left.(\mathbf{u} \cdot \mathbf{v})\right|_{\mathrm{s}_{\mathrm{u} \sigma}}=0,\left.\quad(v \cdot \mathbf{T} \cdot \boldsymbol{\tau})\right|_{\mathrm{s}_{\mathrm{u} \sigma}}=0, \quad M \in \mathrm{S}_{\mathrm{u} \sigma}
$$

where $\mathrm{S}_{\sigma}, \mathrm{S}_{\mathrm{u} \sigma},-$ surfaces for which displacements, forces and mixed (contact) boundary conditions are given; $\boldsymbol{T}$ - stress tensor; $\boldsymbol{F}(M)$ - surface force vector given for surface $\mathrm{S}_{\sigma} ; \boldsymbol{v}, \boldsymbol{\tau}$ - single vectors that determine the normal and tangential directions to surface $S$ at point $M$.

A SSS analysis was carried by the method of MSC.PATRAN modelling. The specimens and their SSSs are symmetric with respect to the planes of symmetry of the U-shaped grooves; therefore, to reduce the number of computing resources, their fourth part was discretized and boundary conditions for kinematic fixation were set. Finite element (FE)-models were digitized on the basis of the first-order hexahedron. The method of controlled localization described in (Zenkov et al. 2011) was used. The FE grid was condensed to the working zone on the lower groove surface following the law of progression (see Fig. 2). The total number of FE did not exceed 110000. The discrete model is shown in Fig. 2.

On the outer edge of the bearing surfaces, boundary conditions corresponded to the axial fixation of this edge. The design loading was carried out by applying a unit pressure uniformly distributed within the circle of a small diameter to the central part of the specimen. This diameter was equal to a quarter of the diameter of the upper surface of the specimen. The specimen fixation conditions are shown in Fig. 3. Under the elastic deformation, structural steel was used: Young's modulus $E=210,000$ $\mathrm{MPa}$, Poisson's ratio $v=0.3$, mass density $\rho=7,85 \mathrm{E}-9$ tons $/ \mathrm{m}^{3}$. 


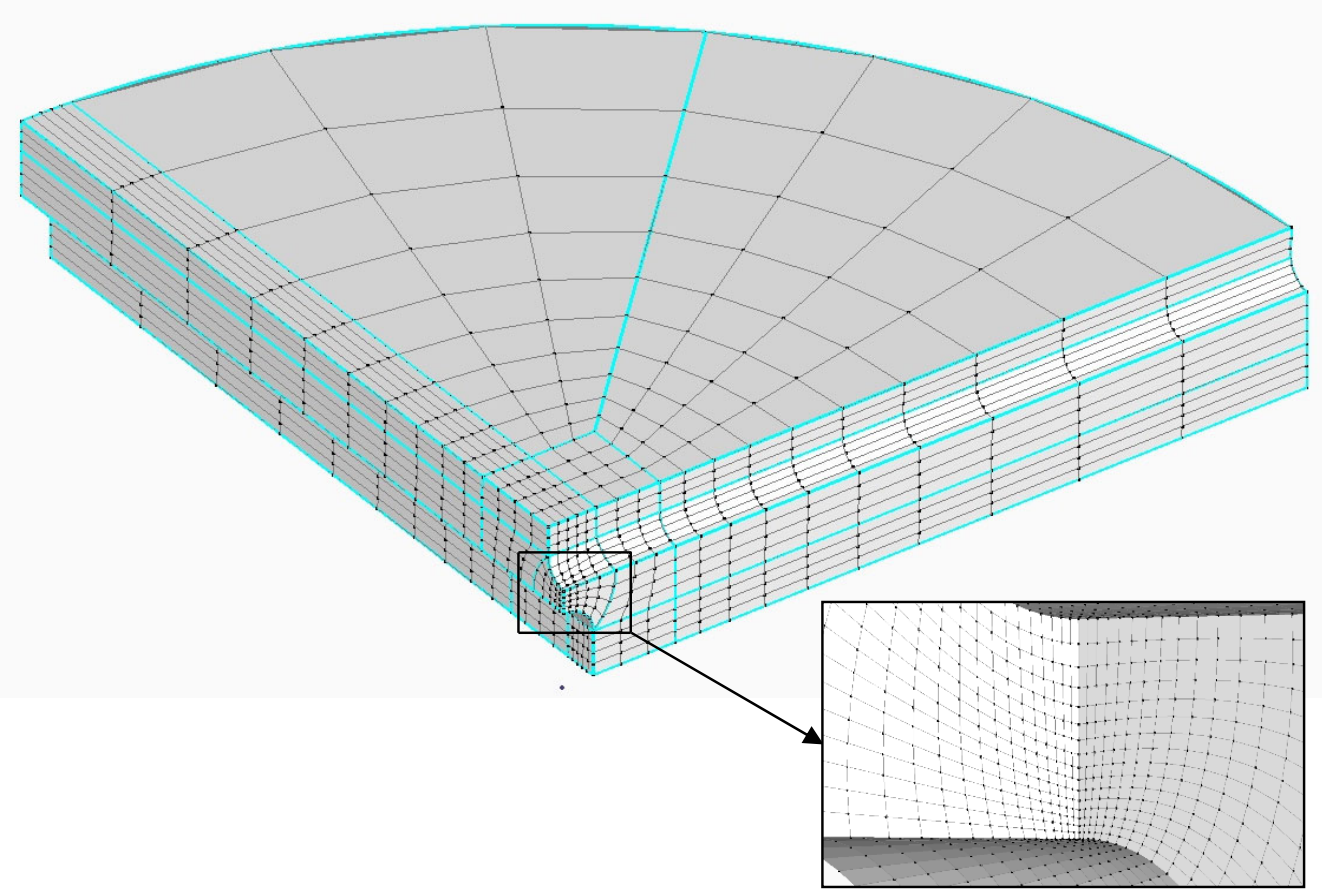

Fig. 2. Discretization of the FE model
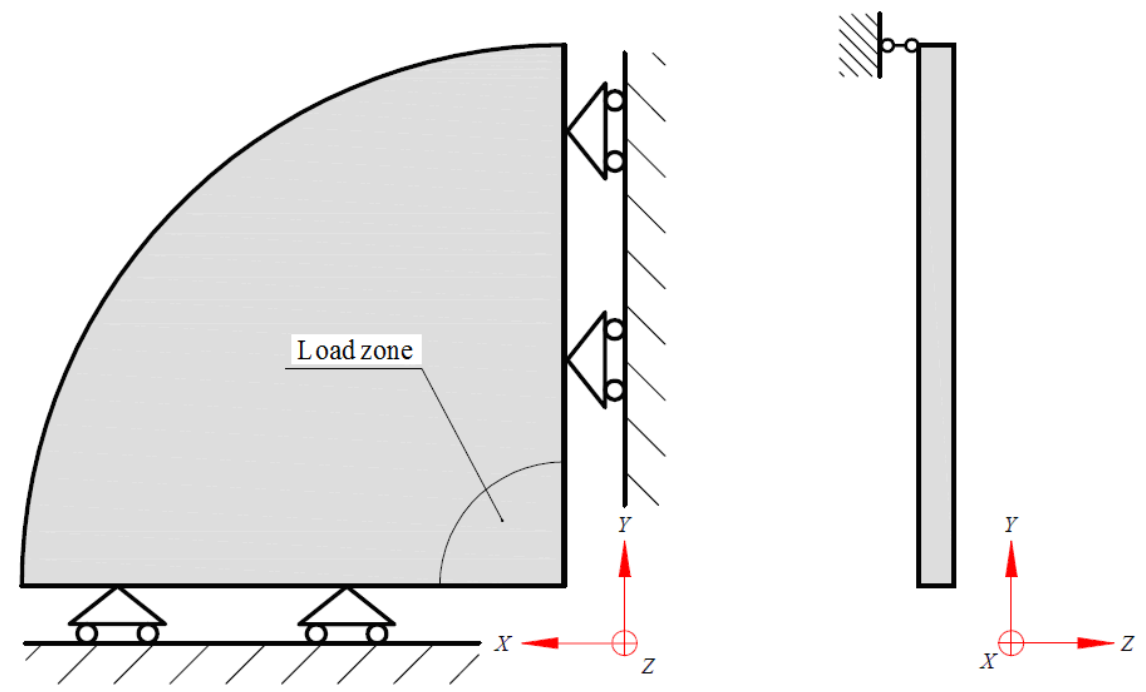

Fig. 3. Disc specimen fixation conditions.

The initial geometric parameters are the width and depth of the lower and upper grooves. The dependences of $P$ calculated by formula (1) and $\mathrm{K}_{\sigma}$ on the geometrical parameters were built for the working zone. The coefficient of concentration of equivalent stresses $\mathrm{K}_{\sigma}$ was calculated by formula,

$$
K_{\sigma}=\frac{\sigma_{i}^{\max }}{\sigma_{i}^{\text {ном }}}
$$

where $\sigma_{i}^{\max }$ - equivalent stresses at the point of observation of the working zone; $\sigma_{i}^{\text {nom }}$ - nominal equivalent stresses - stress intensity in the center of the similar (by diameter and thickness) disc specimen without concentrator grooves. The outer diameter was invariable and equal to $200 \mathrm{~mm}$, thickness $\mathrm{H}=20 \mathrm{~mm}$. 


\section{Results of numerical simulation of SSS of disc specimens}

Structural variants of the disc specimens were used to simulate the values of $\mathrm{K}_{\sigma}$ and $\mathrm{P}$ that are characteristic of real structures. As a rule they are made from materials that are elastically deformed under the operation conditions, and $K_{\sigma}$ is no more than 4 (Birger et al., 1979; Kuznetsov et al., 1999). The following ranges of variable dimensionless values of geometric parameters were studied (Fig. 4),

$$
\rho_{\mathrm{н}}=\frac{R_{H}}{H} \in[0,25 ; 0,6] ; \rho_{o}=\frac{R_{O}}{H} \in[0,15 ; 0,25] ; \zeta=\frac{Z}{H} \in[-0,2 ; 0,15] .
$$

$H$ - total thickness of the specimen; $R_{l}$ - groove radius on the load side, $R_{b}$ - groove radius on the bearing side, $Z$ - distance from the observation point to the middle surface of the original circular disc (without concentrator grooves) taken with a minus sign if the observation point is below the middle and with a plus sign, if it is above the middle surface. The following dimensionless geometric parameters were analyzed: $\rho_{1}$ - relative groove radius on the load side; $\rho_{o}-$ relative groove radius on the bearing side; $\zeta$ - relative displacement of the observation point from the median surface of the original disc.

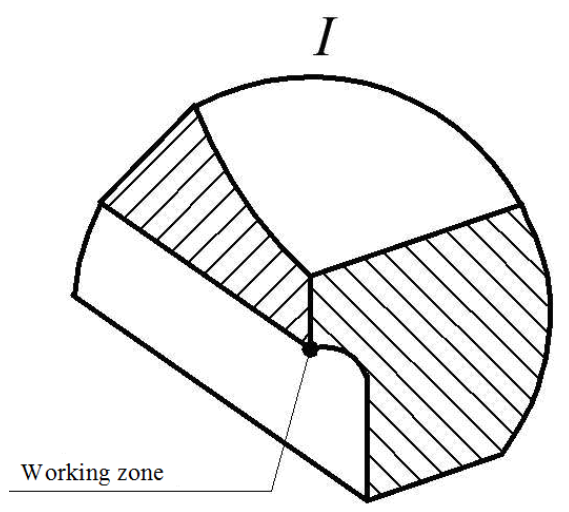

Ring pivot bearing

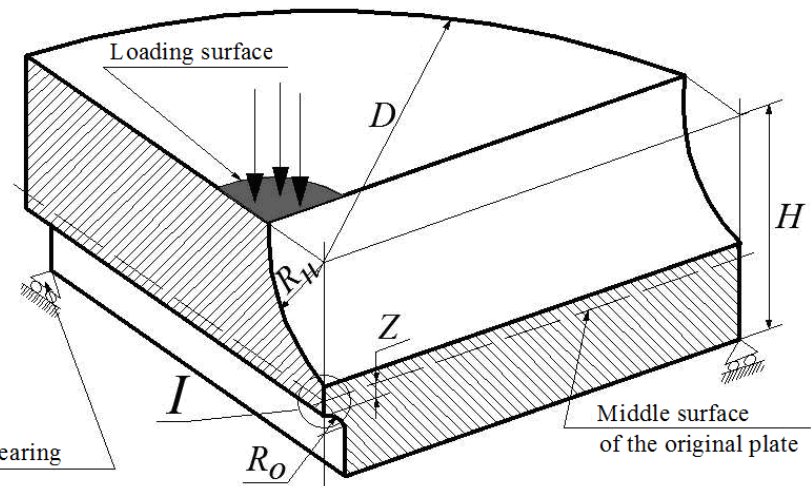

Fig. 4. Geometric parameters of the disc specimen

(I) Working zone of the specimen.

Fig. 5 shows the results of a FE analysis as a distribution of equivalent Von Mises stresses $\sigma_{i}$ for one of the specimen options.

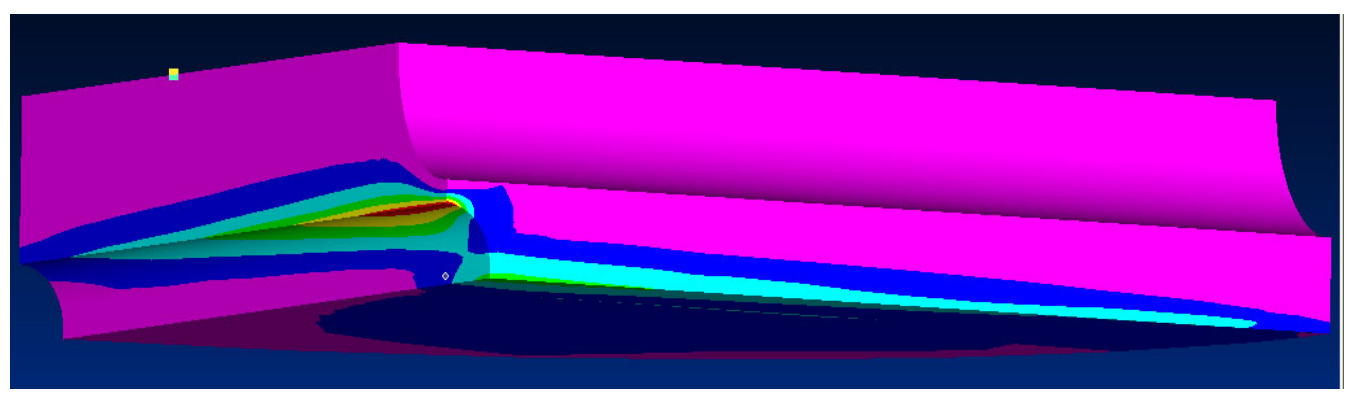

Fig. 5. Distribution of stress intensity $\sigma_{i}$ in the disc specimen.

Fig. 6 and Fig. 7 show the results of variant studies of the stress-strain state of disc specimens under the elastic volumetric deformation. 


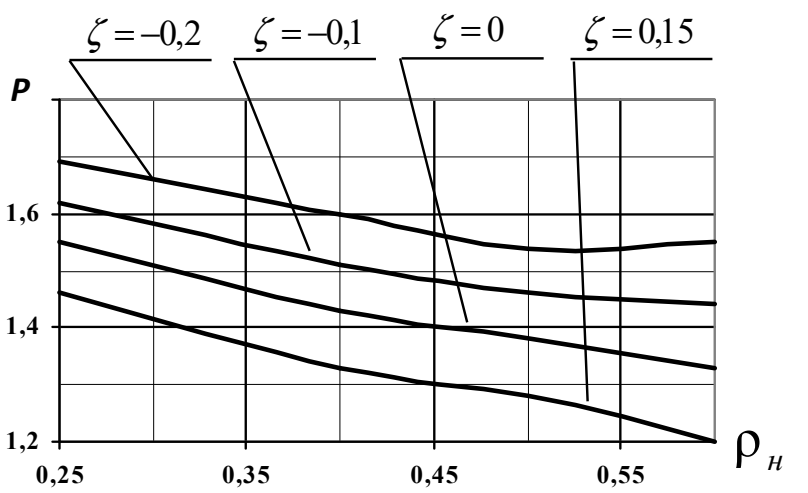

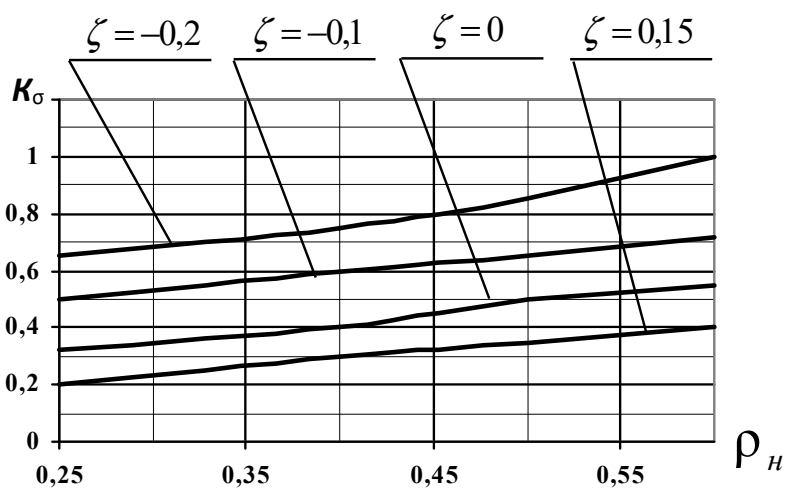

b

Fig. 6. (a) Dependence of coefficient $P$ and (b) $K_{\sigma}$ on the geometric parameters of plane cylindrical specimens at $\rho_{\mathrm{o}}=0,15$

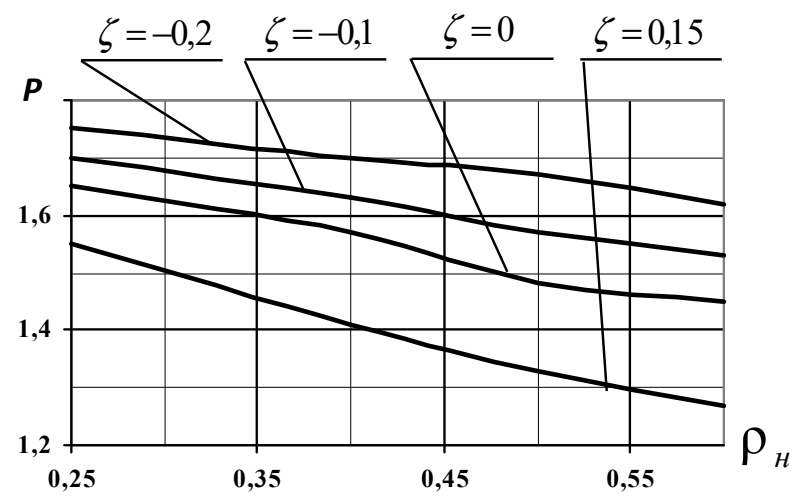

b

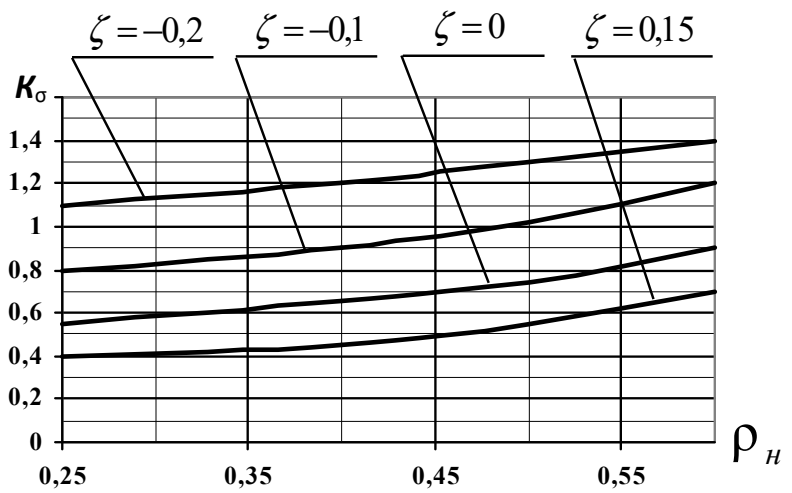

Fig. 7. (a) Dependence of coefficient $P$ and (b) $K_{\sigma}$ on the geometric parameters of plane cylindrical specimens at $\rho_{\mathrm{o}}=0,25$

The distributions of $P$ and $K_{\sigma}$ values in the working zone of the disc specimens at a fixed value of the groove radius on the bearing side $\rho_{o}=\rho_{o}{ }^{\min }=0,15$ and $\rho_{o}=\rho_{o}{ }^{\max }=0,25$ are shown in Fig. 6(a) and Fig. 6(b) and Fig. 7(a) and Fig. 7(b), respectively. The computational experiments show that the value of $P$ varies from 1.2 to 1.75 (Fig. 6 and Fig. 7). Stress concentration $K_{\sigma}$ has a relatively low value (varies within $0.2<K_{\sigma}<1.4$ ). The disadvantage of round specimens revealed by the method of numerical SSS modeling in a wide range of variations in the geometric parameters is due to the fact that variations in groove depths, widths and rounding radii do not change the value of $P$ in the range described by inequalities (3). This range is only partially modeled with disc specimens.

\section{Results of numerical simulation of SSS of disc specimens with truncation}

The strength modelling accuracy improvement task can be solved by numerical modeling of a laboratory specimen that is able to create a range of changes in coefficient $P$ described by inequality (3). Truncated circular disc 1 of a constant thickness (the main disc) with bearing surface 2 and loading surface 5, bounded by external circular contours 3 whose centers coincide with the geometric centers of these surfaces is studied (Fig. 8). This circular disc is equipped with edge notch 4 (groove) located on one of these surfaces and has truncation 6 of the main disc, which is created by two planes symmetric about the geometric center of the main disc and perpendicular to its middle surface (truncation planes). Edge notch 4 is a groove that has a V-shape in the cross-section and is symmetric about the plane perpendicular to the median surface of main disc 1 and passing through the geometric center of this surface. 


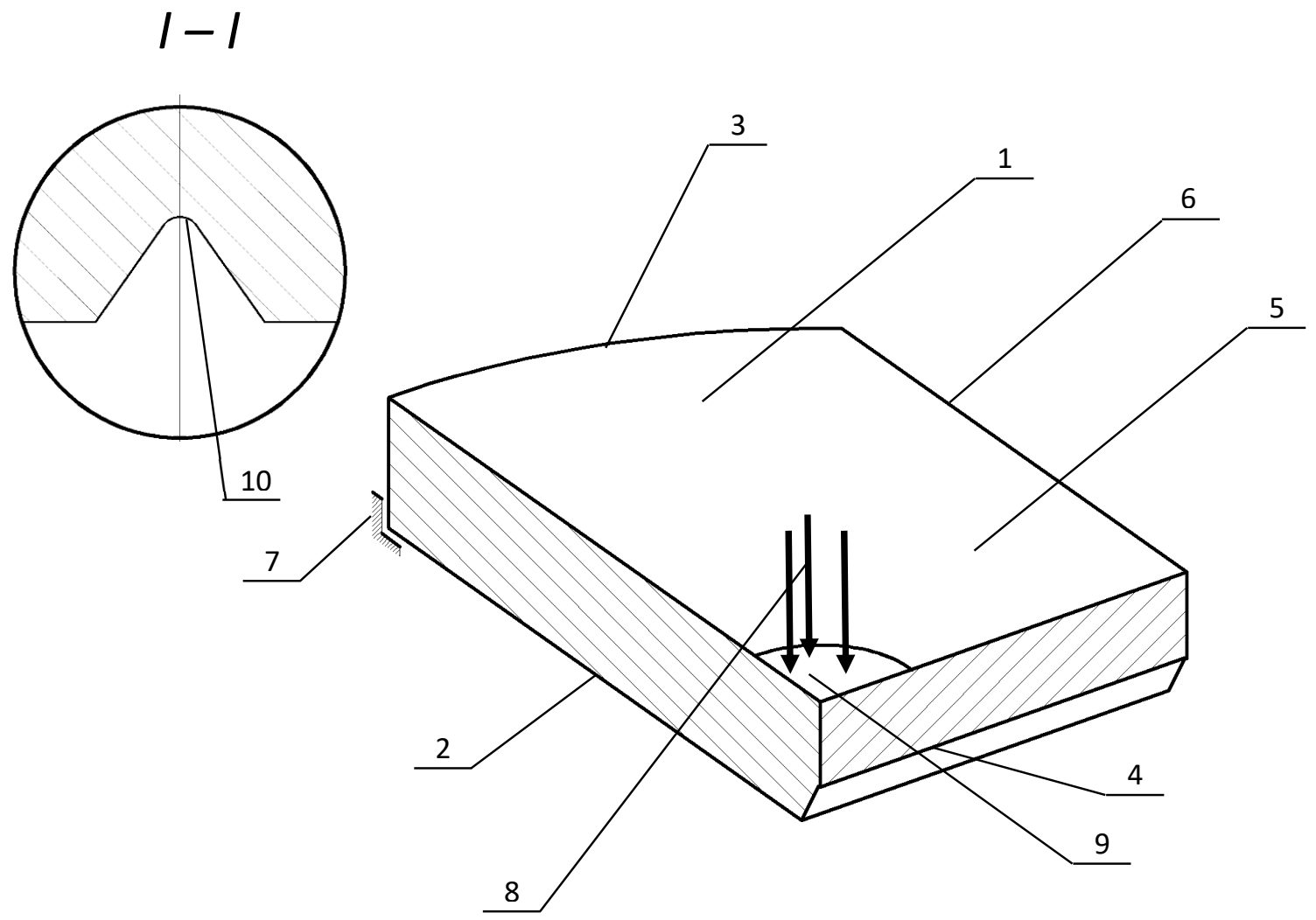

Fig. 8. Structural diagram of the disc specimen

(a quarter of the specimen truncated by two planes of symmetry)

$(I-I)$ Working zone

The disc specimen rests on its outer contour 3 on ring support 7. In the central part of the specimen, transverse test force 8 distributed over the surface of circular shape 9 is applied to loading surface 5 . Under the force applied to the surface of rounding radius 10 , which is the working zone - a concentrator of mechanical stresses - and groove 4 on the axis of rotation, the biaxial stress-strain state occurs.

The disc specimen is deformed when being tested. The specimen is based on its outer contour 3 of bearing surface 2 . Under test force 8 distributed over circular surface 9, disc 1 bends and the bending moment occurs in the working zone of the surface of rounding radius 10 , which causes positive stresses $\sigma_{1}$ - tensile stresses acting perpendicular to groove 4 . Simultaneously, when loading the working zone towards groove 4, the bending moment occurs on the surface of rounding radius 10 , which causes positive stresses $\sigma_{2}$ - tensile stresses acting on groove 4 . Stresses $\sigma_{3}$ on the surface of rounding radius 10 are equal to 0 , which creates the biaxial SSS on the surface of rounding radius 10 . The task of a FE analysis is to check the possibility of modeling the SSS that occurs in the working zone of the specimen in the range (3) by changing the distance between two truncation planes. The specimen is characterized by a number of geometric parameters. In computational experiments, the following ranges of variation in variable dimensionless values of the geometric parameters were studied (Fig. 9),

$$
k=\frac{2 S}{D} \in[0,1 ; 1] \text {. }
$$

In Fig. 8, $N$ is the total thickness of the specimen, $H=20 \mathrm{~mm}, r$ is the groove radius on the bearing side, $r=3 \mathrm{~mm}, S$ is the distance between the geometric center of the main disc and one of the specimen truncation planes, $h_{0}$ is the groove height on the bearing side, $h_{0}=10 \mathrm{~mm}$. In accordance with equality (8), $k$ is the degree of specimen truncation. Fig. 10 shows a distribution of equivalent Von Mises stresses $\sigma_{i}$ for one of the disc specimens. The kinematic specimen fixation conditions are in line with Fig. 3. 


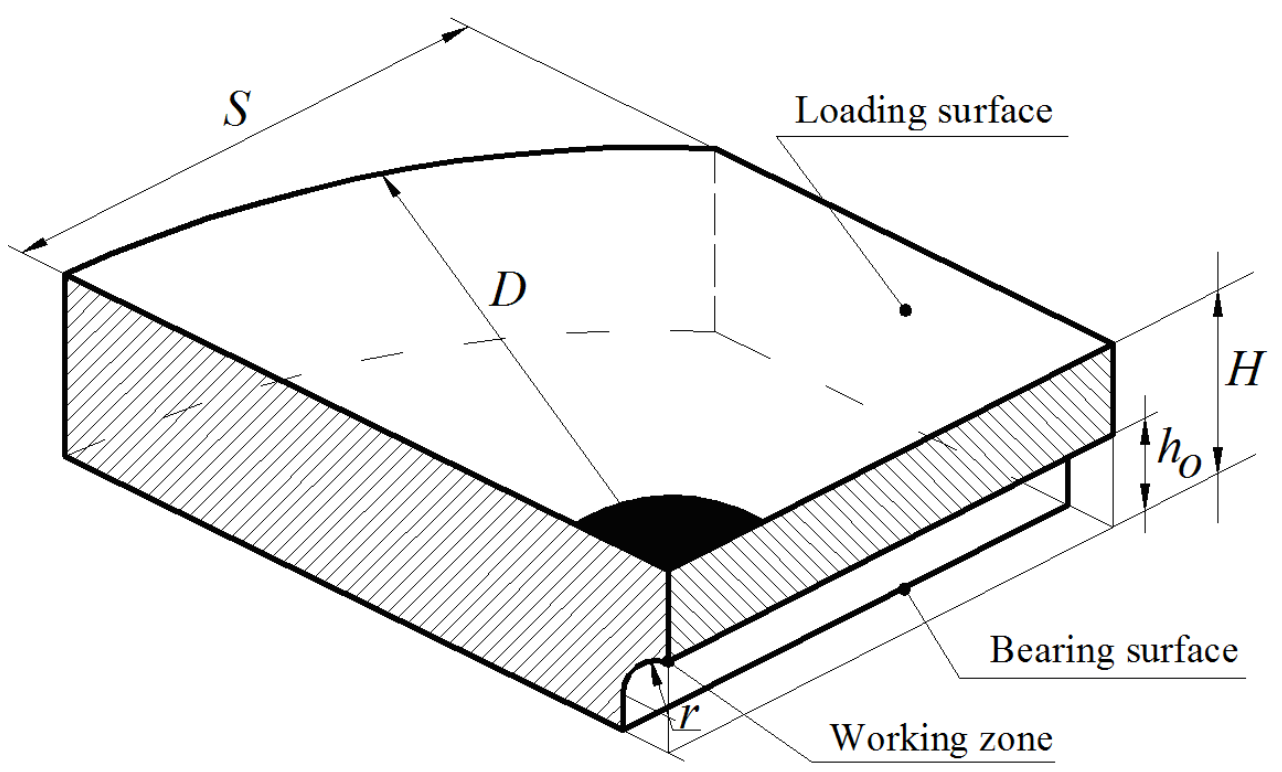

Fig. 9. Geometric parameters of the disc specimen

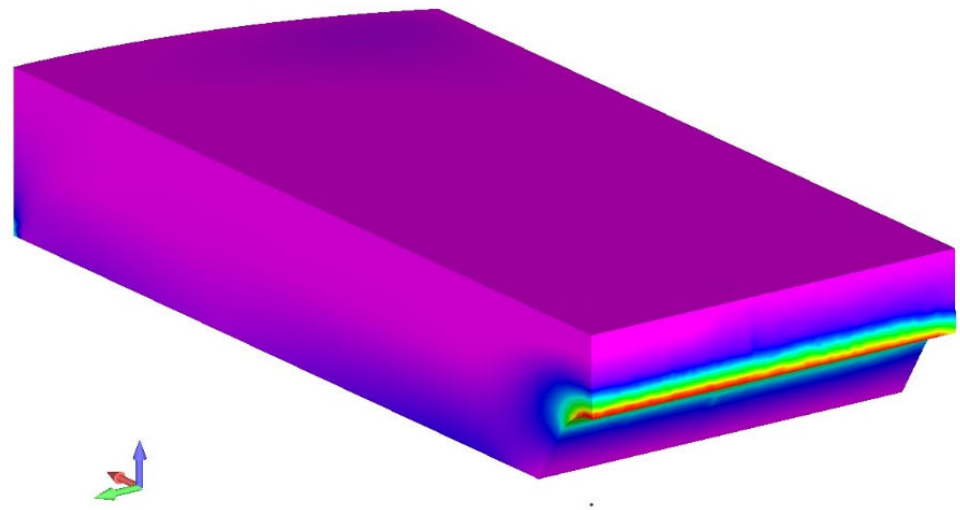

a
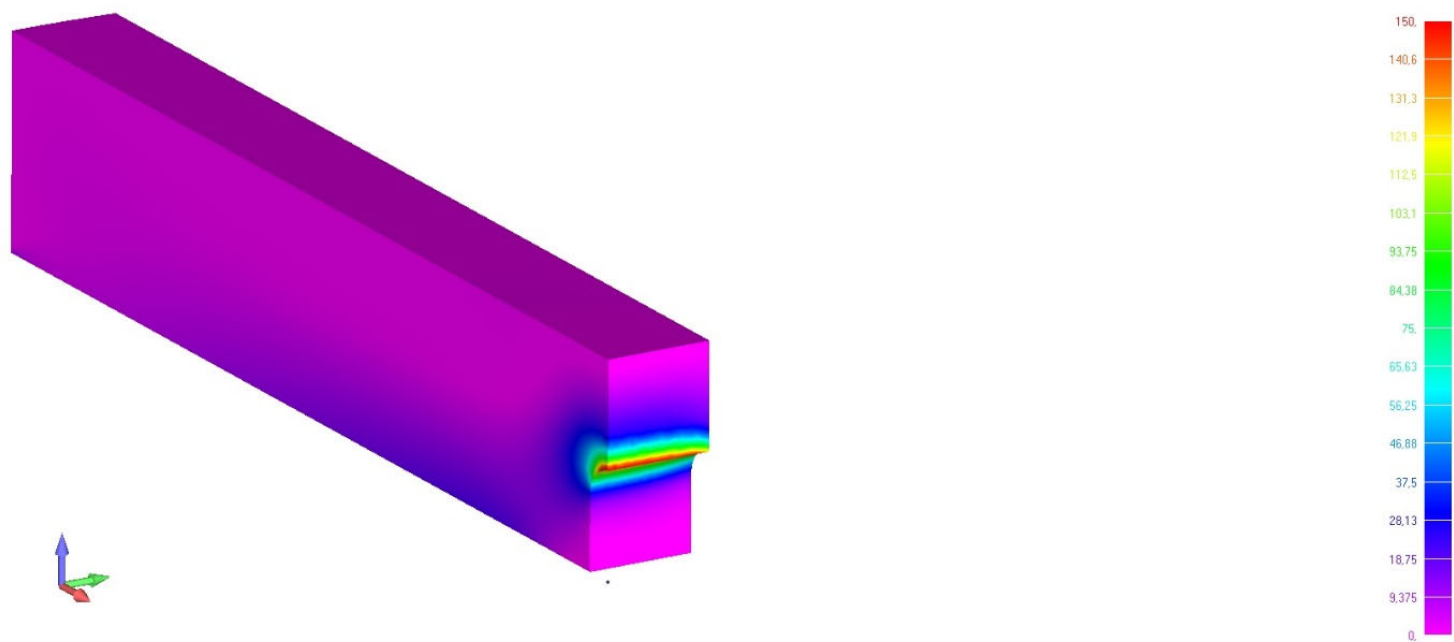

b

Fig. 10. Stress intensity distribution $\sigma_{i}$ in the truncated disc specimen (a) at $k=0.8$ and (b) at $k=0.1$ 
The SSS simulation results have shown that in the working zone stress intensity $\sigma_{i}$ is maximum. For this reason, the destruction site is localized in the middle part of the specimen on the surface of rounding radius 10 (in the working zone of the disc specimen shown in Fig. 8). Fig. 11 shows the dependence of coefficient $P$ on $\mathrm{k}$ - the relative truncation of the disc specimen for the total thickness $\mathrm{H}=20 \mathrm{~mm}$, the groove radius on the bearing side $\mathrm{r}=3 \mathrm{~mm}$, the groove height on the bearing side $\mathrm{h}_{0}=10 \mathrm{~mm}$ and the diameter $\mathrm{D}=200 \mathrm{~mm}$. Figure 11 shows that when varying the values by truncating the disc specimen (S in Fig. 9), $P$ can have any value that satisfies one of the inequalities (3).

When simulating biaxial stress states (the first inequality (3)), the required type of SSS is created by changing the position of the specimen in the testing machine: the loading surface and the bearing surface interchange, and the specimen is turned over.

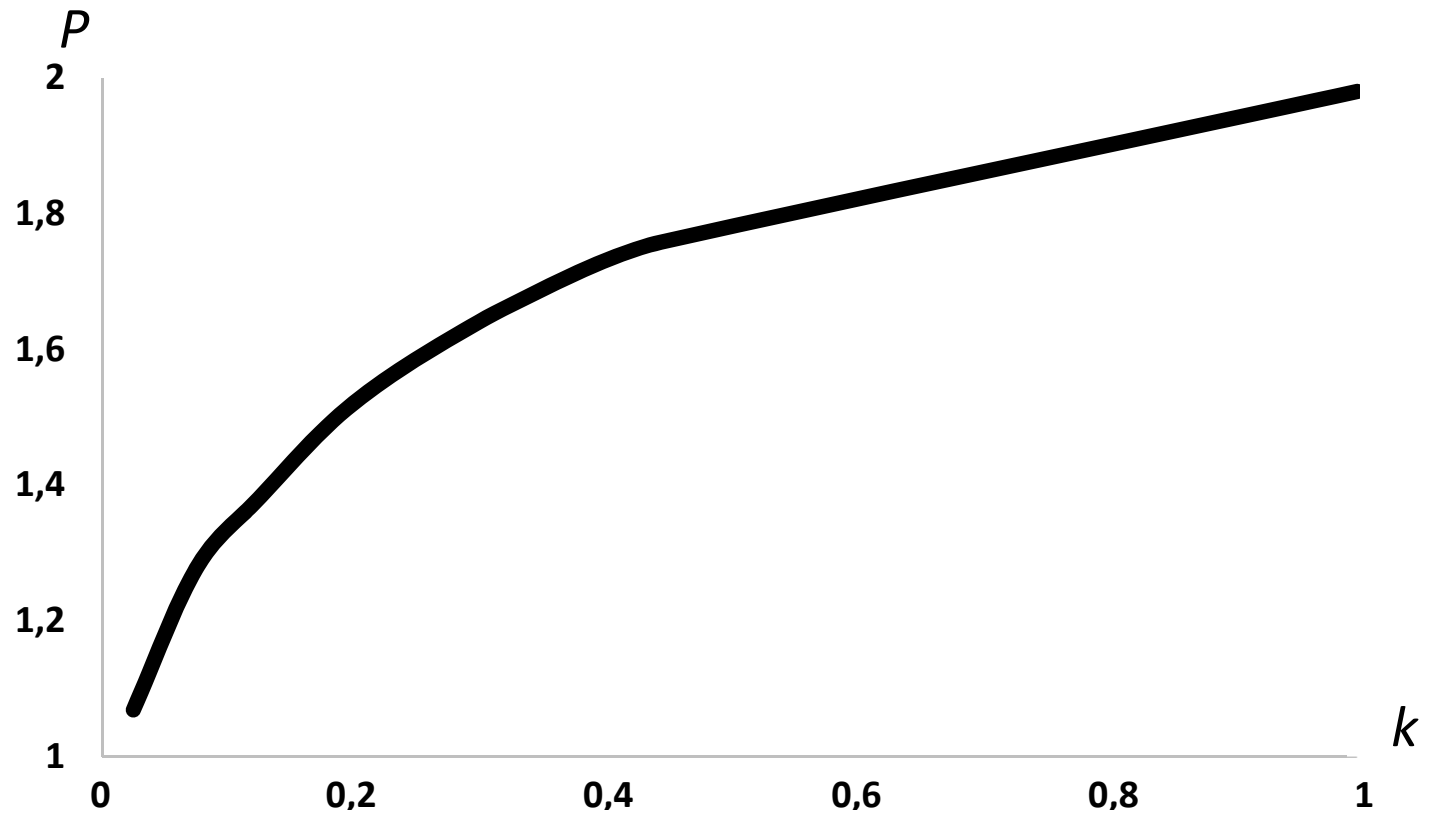

Fig. 11. The $P$ to $k$ ratio with invariable parameters $H, r, h_{0}$ and $D$

Fig. 11 shows that in order to vary the value of coefficient $P$ in the working zone corresponding to inequalities (3), it is sufficient to change the degree of truncation.

\section{Conclusion}

1. Disc specimens improve the accuracy of assessment of the structural strength of materials tested on standard singledrive testing machines with regard to the real type of stress-strain state that occurs in the bearing elements. At the destruction site, the SSS varies in a small range.

2. It has been numerically shown that truncation of a disc specimen can change the type of stress-strain state that occurs in the working zone. Therefore, these specimens can be used to assess the structural strength of materials on standard onedrive testing machines with regard to the SSS type. The numerical SSS modeling allowed us to build dependences of the SSS types on the disc specimen truncation and choose geometric parameters of the specimens to assess the strength of materials.

3. For the disc specimen, the variation in the relative truncation makes it possible to simulate various ratios of biaxial tension, including the biaxial SSS, characterized by the maximum value of coefficient $P\left(\mathrm{P}_{\max }=2\right)$. This makes it possible to apply the specimens for assessing the static and fatigue strength of the material of load-bearing elements under the complex stress-strain state characterized by inequalities (3).

\section{References}

Birger, I.A., Shorr, B.F., \& Ioselevich, G.B. (1979). Calculation of the strength of machine parts. M.: Mechanical Engineering. GosNIIV, R. (1996). Standards for the calculation and design of wagons of $1520 \mathrm{~mm}$ gauge railways of the Ministry of Railways (non-self-propelled). M.: VNIIZhT.

GOST 25.504-82. (2004). Calculations and strength tests. Methods for calculating the characteristics of fatigue resistance. M., IKP Publishing house of standards,

Kogaev, V.P., Makhutov, N.A., \& Gusenkov, A.P. (1985). Calculations of machine parts and structures for strength and durability. Moscow: Machine building.

Kuznetsov, A.M., Livshits, V.I., Tatarinov, V.G. \& et al. (1999). Vessels and high-pressure pipelines: Handbook. Irkutsk: 
Irkutsk Regional Printing House.

Lebedev, A.A., Kovalchuk, B.I. \& [et al.] (2003). Mechanical properties of structural materials in a complex stress state. Kiev: In Yure.

Pisarenko, G.S., Yakovlev, A.P., \& Matveev V.V. (2008). Handbook on resistance of materials. Kiev: Delta.

Smirnov-Alyaev, G.A. (1968). Mechanical foundations of plastic processing of metals. Engineering methods. L.: Mechanical Engineering.

Tsvik, L.B., Pimshtein, P. G., \& Borsuk, E.G. (1978). Experimental studies of the stress-strain state of a multilayer cylinder with a monolithic bushing. Problems of strength, 4, 74-77.

Tsvik, L.B., \& Zenkov, E.V. (2015). Determination of strength characteristics of materials of experimental prismatic specimens under the biaxial tension. Bulletin of machine building,1, 42-46.

Tsvik, L.B. (2017). Specimens for mechanical testing of structural steels under cyclic loading. Transport infrastructure of the Siberian region, 2, $834-839$.

Tsvik, L.B., Zenkov, E.V., Bocharov, I..S., \& Elovenko, D.A. (2020). Disc specimen for assessing the structural strength of the material. Patent RF No.2734276, dated 14.10.2020, Bul. No. 29.

Tsvik, L.B., Pykhalov, A.A., Khramenok, M.A. \& et al. (2009). Specimen for assessing the strength of a material in a complex stress state. Patent RF No.2360227, dated 27.10.2009, Bul. No. 18.

Vilimok, Ya.A., Nazarov, K.A., \& Evdokimov, A.K. (2013). Stress state of flat specimens under the uniaxial and biaxial tension. News of TSTU. Technical science, 11, 388 - 39.

Zenkov, E.V., \& Tsvik, L.B. (2015). Formation of divergent testing efforts and experimental evaluation of material strength under biaxial stretching. PNRPU Mechanics Bulletin, 4, 110-120.

Zenkov, E.V., \& Tsvik, L.B. (2018). Accuracy improvement for combined static strength criterion for structures under complex loading. Materials Physics and Mechanics, 40, 124-132.

Zenkov, E.V. (2017). Update of the equations of the limit state of the structural material with the realization of their deformation. Journal of Physics: Conference Series, 944, 012128.

Zenkov, E.V., \& Tsvik, L.B. (2017). Refinement of the equations of the limiting state of the structure material taking into account the real type of their deformation. Systems. Methods. Technology, 2(34), 28-34.

Zenkov, E.V., Aistov, I.P., \& Vansovich, K.A. (2019). Modeling stress state stiffening of the nozzle zone of pressure vessel by the finite element method. AIP Conference Proceedings, 2141, 030042.

Zenkov, E.V., Tsvik, L.B., Pykhalov, A.A. \& et al. (2014). Prismatic specimen for assessing the strength of the material. Patent RF No.2516599, dated 20.09.2014, Bul. No. 14.

Zenkov, E.V., Tsvik, L.B., \& Pykhalov, A.A. (2011). Discrete modeling of the stress-strain state of flat-cylindrical specimens with grooves as stress concentrators. Bulletin of Irkutsk State Technical University, 7(54), 6-11.

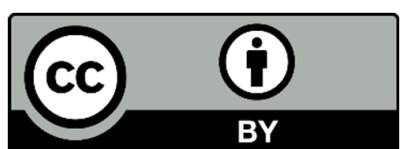

(C) 2022 by the authors; licensee Growing Science, Canada. This is an open access article distributed under the terms and conditions of the Creative Commons Attribution (CC-BY) license (http://creativecommons.org/licenses/by/4.0/). 